\title{
Decoding dangerous death: how cytotoxic chemotherapy invokes inflammation, immunity or nothing at all
}

\author{
RG van der Most ${ }^{1}$, AJ Currie ${ }^{1}$, BWS Robinson ${ }^{1}$ and RA Lake ${ }^{\star, 1}$
}

Chemotherapy and immunotherapy can be either synergistic or antagonistic modalities in the treatment of cancer. Cytotoxic chemotherapy not only affects the tumor but also targets dividing lymphocytes, the very cells that are required to develop an immune response. For this reason, chemo- and immunotherapy have been seen as antagonistic. However, cell death can be immunogenic and the way in which chemotherapeutic drug kills a tumor cell is likely to be an important determinant of how that dying cell interacts with the immune system and whether the interaction will lead to an immune response. When a cell dies as the result of infection, the immune system responds rapidly and the system of Toll-like receptors (TLR) plays a key role in this process. In this review, we will briefly summarize the intracellular signaling pathways that link TLR ligation with immune activation and we will address the questions where and how TLRs recognize their targets.

Cell Death and Differentiation (2008) 15, 13-20; doi:10.1038/sj.cdd.4402255; published online 16 November 2007

It is now abundantly clear that chemotherapy and immunotherapy can be either synergistic or antagonistic modalities in the treatment of cancer. Cytotoxic chemotherapy not only affects tumor but also targets dividing lymphocytes, the very cells that are required to develop an immune response. For this reason, chemo- and immunotherapy have been seen as antagonistic. On the other hand, chemotherapy also depletes regulatory $T$ cells and could, in this way, enhance potential immune responses. Furthermore, lymphodepletion triggers homeostatic T-cell reconstitution, creating new populations of pre-T cells that need education in the thymic environment. The post-chemotherapy phase of immune system reconstitution therefore provides a unique opportunity for therapeutic intervention by shaping the repertoire toward reactivity to tumor antigens. Because this has important therapeutic implications, we need to get a clear view of the biological basis that underlies any potential synergy. ${ }^{1}$ Such an understanding may help to explain why most tumor immunotherapy trials might have failed in the past and predict some key requirements for success in the future.

The way in which chemotherapeutic drug kills a tumor cell is likely to be an important determinant of how that dying cell interacts with the immune system and whether the interaction will lead to an immune response. Immunogenic cell death induces dendritic cell (DC) maturation allowing the DC to activate relevant $\mathrm{T}$ cells. In contrast, non-immunogenic cell death is bland and does not activate DC. This was clearly illustrated in a recent study showing that the cytotoxic drug doxorubicin leads to the translocation of calreticulin, a molecular flag that directs dendritic-cell uptake of the flagbearing cells. ${ }^{2,3}$ These findings add to the growing interest in the immunogenicity of cell death and the impact of different therapeutic interventions on the interaction between dying cells and the immune system. ${ }^{1}$

It is perhaps not surprising that the interaction between apoptotic cell death and the immune system is a complex one. On the one hand, billions of cells die by apoptosis every day as part of normal tissue homeostasis, which suggests that this form of apoptosis is non-immunogenic because it does not result in overwhelming autoimmunity. On the other hand, injured or infected cells also undergo apoptotic cell death and cannot be ignored by the immune system. The default response to apoptosis may very well be a tolerogenic one. ${ }^{4}$ The translocation of phosphatidylserine (PS) from the inner to the outer leaflet of the plasma membrane is one important signal that prevents unwanted immune responses. PS favors uptake of apoptotic cells by macrophages rather than dendritic cells and stimulates the production of immunosuppressive cytokines, such as TGF- $\beta$ and interleukin (IL)-10. However, when a cell dies as a result of infection or injury, the immune system must launch an appropriate response. Thus, the immune system must decode the way in which a cell dies and this decoding process leads to the decision of how best to respond. In our view, the decision making process is most easily understood in the context of the danger model, put forward by Matzinger. 5,6 The model asserts that danger

\footnotetext{
${ }^{1}$ University Department of Medicine, National Centre for Asbestos Related Diseases, University of Western Australia, Perth, Australia

${ }^{*}$ Corresponding author: RA Lake, School of Medicine and Pharmacology and Western Australian Institute for Medical Research, University of Western Australia, 4th Floor, G-block, Sir Charles Gairdner Hospital, Nedlands, Perth 6009, Australia. Tel: +61 89346 3127; Fax: +61 89346 2816; E-mail: rlake@ cyllene.uwa.edu.au Keywords: chemotherapy; immunotherapy; inflammation; toll-like receptors; danger signals

Abbreviations: DAMPs, damage-associated molecular patterns; DC, dendritic cell; HMGB1, high-mobility group box 1; HSPs, heat-shock proteins; PAMPs, pathogenassociated molecular patterns; PS, phosphatidylserine; ROS, reactive oxygen species; TLR, Toll-like receptors.

Received 17.7.07; revised 10.9.07; accepted 11.9.07; Edited by G Kroemer; published online 16.11.07
} 
signals include both exogenous pathogen-associated and endogenous damage-associated molecular patterns (PAMPs and DAMPs). It predicts that endogenous signals are equally efficient as exogenous ones at initiating immune responses.

\section{Cell Death and Infection}

When a cell dies as a result of infection, the immune system must respond rapidly. The system of Toll-like receptors (TLR) plays a key role in this process. ${ }^{7}$ TLRs recognize PAMPs such as double-stranded RNA, unmethylated DNA containing CpG-motifs, LPS and flagellin. Both in humans and in mice, multiple TLRs recognize nucleic acid structures, whereas the others bind to hydrophobic regions of pathogen-derived molecules, identifying hydrophobicity and nucleic acid as the key structures. ${ }^{6}$ In this review, we will briefly summarize the intracellular signaling pathways that link TLR ligation with immune activation and we will address the questions where and how TLRs recognize their targets. ${ }^{8}$ It has become clear during the last few years that endogenous ligands activate the same signaling pathways as exogenous ligands through the TLR system. These pathways are therefore critical to the decision making process associated with tissue injury (Figures 1-3). A full list of the key molecular players, as shown in the figures, and their abbreviations is provided in Box 1.

With the exception of TLR-3, TLR activation is relayed via MyD88 ${ }^{9}$ (Figures 1 and 2 ). The subsequent signaling cascade involves TRAF6 and IKK and leads to activation of NF- $\kappa \mathrm{B}$ and expression of pro-inflammatory cytokines (IFN- $\beta$, IL-12, TNF- $\alpha$ ). TLR3 ligation drives the expression of a set of genes (IFN- $\beta$, IP-10, RANTES) via a parallel MyD88-independent pathway involving TRIF, TBK, IKK $\varepsilon$ and the IRF3/7 transcription factors. The novel vascular disrupting agent DMXAA appears to be a direct TBK activator, thereby driving IFN- $\beta$ expression. ${ }^{9}$

\section{Box 1}

\begin{tabular}{|c|c|}
\hline $\begin{array}{l}1 \text { TLR2/4/ } \\
7 / 9\end{array}$ & Toll-like receptors \\
\hline 2 MDA5 & Melanoma differentiation associated gene-5 \\
\hline 3 RIG-1 & Retinoid-inducible gene 1 \\
\hline 4 MyD88 & Myeloid Differentiation primary response gene 88 \\
\hline 5 TRIF & TIR-domain containing adaptor inducing IFN- $\beta$ \\
\hline 6 IPS-1 & IFN- $\beta$ promoter stimulator 1 \\
\hline 7 ROS & Reactive oxygen species \\
\hline 8 L-PAM & Melphalan \\
\hline 9 DMXAA & $\begin{array}{l}\text { 5,6-Dimethylxanthenone-4-acetic acid, a vascular } \\
\text { disrupting agent }\end{array}$ \\
\hline 10TRAF6 & TNF receptor associated factor 6 \\
\hline 11IRAK1/4 & IL-1 receptor associated kinase $1 / 4$ \\
\hline 12MAPK & Mitogen-activated kinase \\
\hline 13ASK & Apoptosis signal-regulating kinase \\
\hline $14 p 38$ & protein 38, an MAPK \\
\hline $15 \mathrm{IKK} \alpha \beta \gamma$ & I B kinase $\alpha \beta \gamma$ \\
\hline 16TBK & Tank-binding kinase \\
\hline $17 \mathrm{IKK} \varepsilon$ & IkB kinase $\varepsilon$ \\
\hline 17TANK & TRAF family member associated NF- $\kappa$ B activator \\
\hline $19 I_{\kappa} \mathrm{B}$ & Inhibitor of NF- $\kappa \mathrm{B}$ \\
\hline $20 N F-\kappa B$ & Nuclear factor $\kappa \mathrm{B}$ \\
\hline 21AP1 & Activating protein 1 \\
\hline 22IRF3 & Interferon response factor 3 \\
\hline 23IRF7 & Interferon response factor 7 \\
\hline
\end{tabular}

Overview of signaling factors involved and abbreviations used. Key factors are indicated in bold.

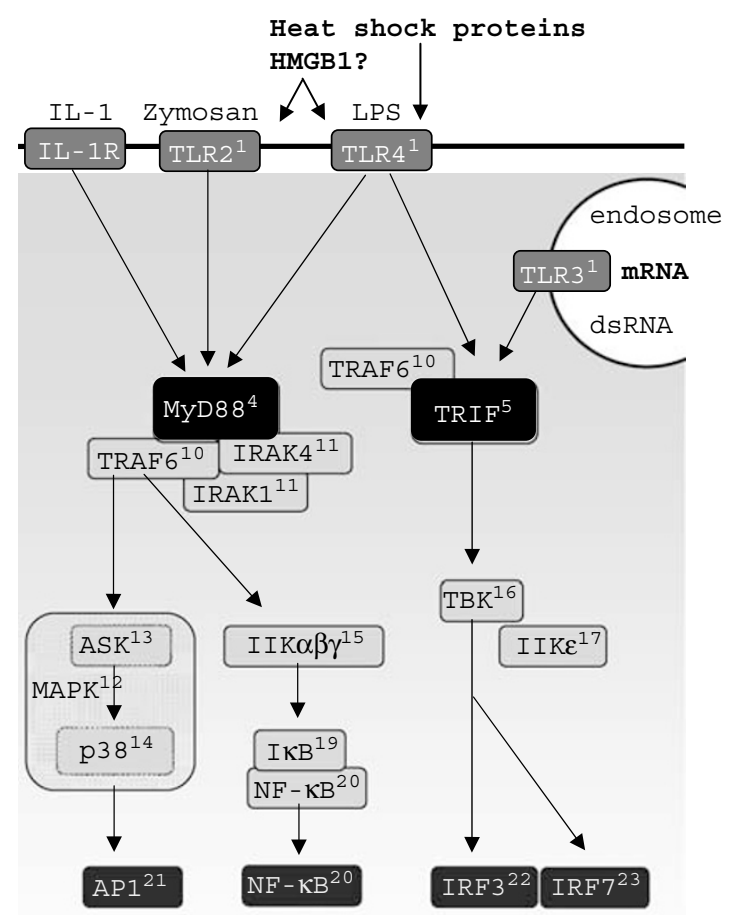

Figure 1 Schematic representation of PAMP and DAMP recognition. Pattern recognition receptors are indicated (no. 1), as well as adaptor molecules (nos. 4-5) downstream signaling molecules (nos. 9-20) and transcription factors (nos. 21-23). Dendritic cell (DC) signaling in response to IL-1R, TLR2, TLR3 and TLR4. TLR ligands and the endogenous danger molecules HMGB1 and HSPs are indicated. See Box 1 for abbreviations (see online version for color figure pattern recognition receptors in red, adaptor molecules in black, signalling molecules in light blue and transcription factors in dark blue).

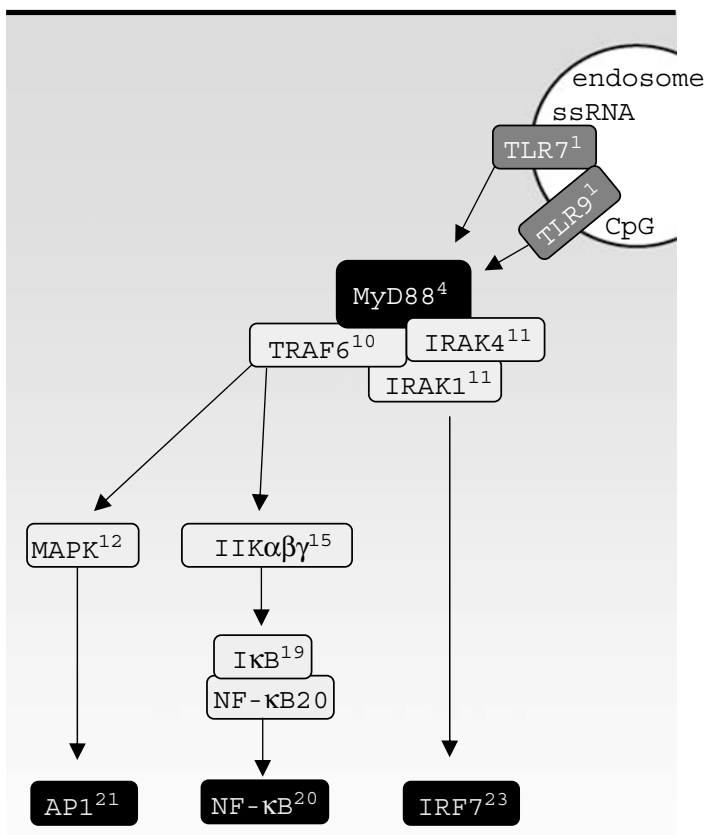

Figure 2 TLR7 and TLR9 responses in plasmacytoid DCs. TLR7/9 and their endosomal ligands and downstream signaling pathways are indicated. Numbering of factors is as in Figure 1 


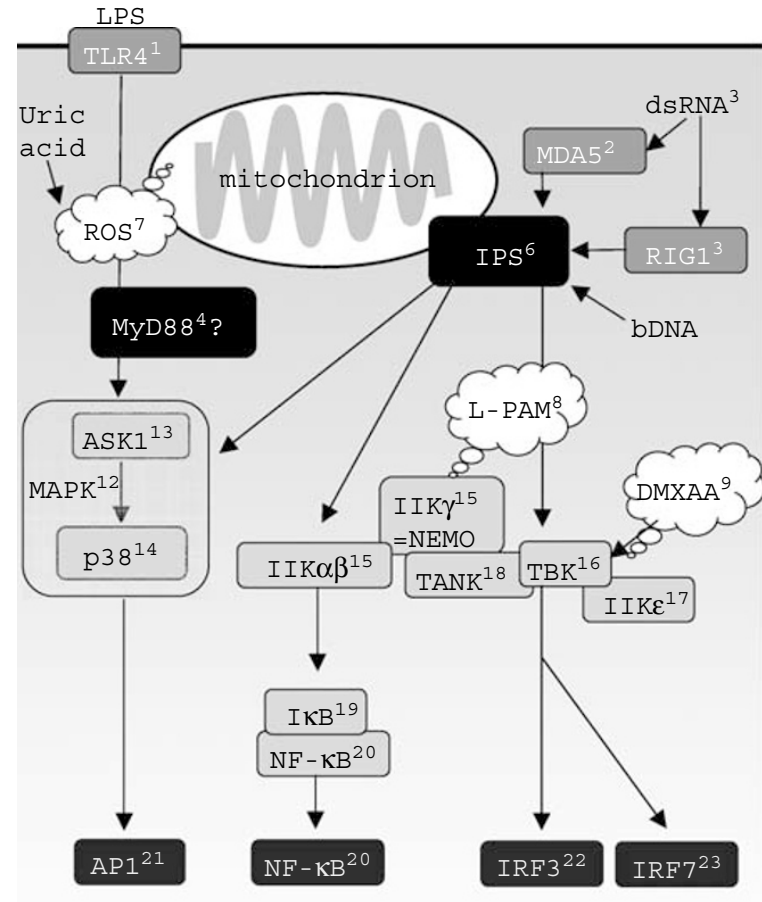

Figure 3 Schematic representation of endogenous danger stimuli (nos. 7-9) as shown in clouds. Involvement of ROS (no. 7) in the response and association with mitochondrial apoptosis; responses to B-form DNA (bDNA), double-stranded RNA, melphalan (LPAM, no. 8) and the vascular disrupting agent DMXAA (no. 9). Numbering and coloring of adaptor molecules (no. 4 and 6), signaling molecules (nos. 9-20) and transcription factor (nos. 21-23) are as in Figure 1 and Box 1

The LPS receptor TLR4 activates pro-inflammatory gene expression via the MyD88-dependent and TRIF-dependent pathways. LPS also activates the IRF3 transcription factor through the MAP kinase pathway, with a central role of ASK1 ${ }^{10,11}$ ASK1 is a pro-apoptotic MAPK kinase kinase that activates the MAPK kinase p38 in response to oxidative stress, anticancer drugs and TNF- $\alpha$. Importantly, ASK1 activation is critically dependent on reactive oxygen species (ROS) (Figure 3). It is likely that ROS-induced dissociation of the ASK1 inhibitor (and redox sensor) thioredoxin plays a key role, allowing TRAF6-ASK1 binding. Combined, these data identify ROS as a potential intracellular messenger of inflammation. However, there is some controversy whether the ASK1-p38 pathway is required for MyD88-dependent or -independent gene expression. ${ }^{10,11}$

The nucleic acid-recognizing TLRs are located in the endosomes of dendritic cells, macrophages and some tumor cells. So how does a DC-based TLR recognize viral or bacterial DNA in an infected apoptotic cell? An elegant series of experiments by Reis e Sousa showed that double-stranded RNA contained in apoptotic cells is taken up by DCs through the endo-phagosomes, resulting in TLR-3 ligation in the endosome, followed by DC activation. ${ }^{12}$ It is possible that the other endosomal TLRs function in a similar way. Thus, when DCs phagocytose apoptotic cells, they decode the viral or microbial content by TLR-mediated pattern recognition in the endosomes. However, recognition of viral nucleic acid is not limited to TLRs. Viral double-stranded RNA is also recognized by RIG-1 and by the PKR system. RIG-1 most likely functions as a viral sensing molecule in infected cells, that is linked to TBK1 via the IPS-1 adaptor molecule. ${ }^{13}$ Activation of these proteins results in type-I IFN production in infected cells through IRF3/7 activation.

TLR ligation can be directly cytotoxic. TLR2 or TLR4 activation in macrophages can trigger apoptosis via the MyD88/caspase-8 or TRIF/mitochondrial death pathways, respectively. ${ }^{14}$ This pathway is likely to be evolutionarily important since it allows the host to rapidly kill microbeinfected macrophages. TLRs are also expressed on tumor cells, and it has been shown that TLR3 ligation on different breast cancer cells results in apoptotic cell death in a TRIF and caspase-8-dependent manner; IFN- $\beta$ is necessary but not sufficient for the induction of apoptosis. ${ }^{15}$ However, not all TLR-3 + tumor cells are killed by exposure to dsRNA. The situation is more complex for TLR2 and TLR4, in that some tumors display accelerated growth after TLR signaling. ${ }^{16,17}$

\section{TLR-Dependent and TLR-Independent Endogenous Danger Signals}

Several endogenous DAMPs have now been identified. ${ }^{18,19}$ Some of these are independent of the TLR system. However, their signaling pathways intersect with the pathways of TLR signaling described above. ${ }^{8}$ Here, we will discuss the roles of mammalian DNA, high-mobility group Box 1 (HMGB1), heatshock proteins (HSPs), uric acid and ROS as endogenous triggers of innate immunity. Other reviews discuss some of the additional potential endogenous damage ligands. ${ }^{18,19}$

The key observation that linked mammalian DNA with innate immunity was that DNasell-deficient macrophages accumulate DNA from phagocytosed cells and produce IFN- $\beta$, TNF- $\alpha$ and IP-10 in a TLR-, MyD88- and TRIF-independent fashion $^{20}$ (Figure 3). DNasell-deficient embryos die in utero because of IFN- $\beta$-induced anemia. Other investigators have shown that the $\mathrm{B}$ form of double-stranded DNA activates IRF3-mediated gene expression (IFN- $\beta$, IP-10) through TBK or NF- $\kappa$ B. ${ }^{21}$ TBK activation depends on RIG-1 human cells, ${ }^{22}$ but is RIG-1 independent in murine cells. ${ }^{21}$ In addition, Stetson and co-workers ${ }^{23}$ recently reported that cytosolic, non-CpG-containing DNA stimulates a non-TLR-dependent IFN- $\beta$ response. This response is not associated with MAPK activation of NF- $\kappa \mathrm{B}$ translocation. Thus, dsDNA from pathogens or injured cells could activate antigen-presenting cells.

The RAX protein may act as a sensor for apoptosisinducing stress, such as chemotherapy, inflammatory cytokines and viral infection. ${ }^{24}$ RAX activates the IFN-inducible dsRNA-dependent protein kinase PKR. PKR activation can lead to $I_{\kappa} \mathrm{B}$ degradation, $\mathrm{NF}-\kappa \mathrm{B}$ activation, IRF-1 expression and elF $2 \alpha$ phosphorylation.

HMGB1 has recently been identified as an endogenous danger signal. ${ }^{25,26} \mathrm{HMGB} 1$ is a nuclear protein and serves as a transcription factor, but has a dual function as a proinflammatory cytokine. The protein is released from necrotic cells and from apoptotic tumor cells when they are killed by $\mathrm{T}$ cells or NK cells. ${ }^{27}$ Activated macrophages, NK cells and mature dendritic cells secrete HMGB1; it promotes lymphocyte traffic across endothelial barriers and induces TNF- $\alpha$, IL-6 
and IFN- $\gamma$ responses, although it is unclear whether this is a direct activity or whether this requires additional cofactors (e.g., single-stranded nucleic acids, LPS). There is considerable debate on the interaction of HMGB1 and the innate immune system, in particular with regard to TLR dependency. Park et al. ${ }^{28}$ have shown that HMGB1 binds both TLR2 and TLR4 (Figure 1), which is consistent with very recently published data showing that immune activation by dying tumor cells involves both HMGB1 and TLR4. ${ }^{29}$ Infact, these authors found a link between post-therapy relapse and a TLR4 loss-of-function allele in breast cancer patients, ${ }^{29}$ highlighting the potential significance of this signaling pathway. In contrast, Tian et al. ${ }^{30}$ show that HMBG1 amplifies CpG-driven responses in a TLR9-dependent and TLR2/4independent manner. These authors identified RAGE (receptor for advanced glycation end-products) as the principal receptor for HMGB1. ${ }^{30}$ Nevertheless, the role of HMGB1 in cancer is still somewhat ambiguous because it promotes chronic inflammation and neoangiogenesis and therefore seems to protect cells from death. ${ }^{25}$ This would be a clear concern, given that HMGB1 is released from chemotherapyor lymphocyte-killed tumor cells. ${ }^{27}$

HSPs have a paradoxical role in tumor cell survival and tumor cell death, ${ }^{31}$ reminiscent to HMGB1. The frequent overexpression of HSP27, HSP70 and HSP90 in tumor cells has an antiapoptotic role and is associated with poor prognosis and resistance against chemotherapy or radiation. ${ }^{31}$ Puzzlingly, however, HSP70 expression in some human tumors is associated with an improved prognosis. In this vein, extracellular HSPs are immunostimulatory, possibly by facilitating cross-presentation of tumor antigens to CD8 T cells, ${ }^{32}$ and by activating DCs via TLR2, TLR4 and CD40 (Figure 1). Indeed, DC stimulation with HSP70 results in the production of pro-inflammatory cytokines (TNF- $\alpha, \mathrm{IL}-1 \beta$, IL-12, IL-6 and GM-CSF). Vaccination with an individualized Gp96 peptide vaccine (Oncophage) has yielded promising results in a Phase II clinical trial in patients with metastatic colorectal cancer. ${ }^{33}$

Crystalline uric acid has recently been identified as an endogenous danger signal. ${ }^{34}$ Soluble uric acid has no adjuvant activity, and may even have anti-inflammatory activity. Uric acid is an end product of purine metabolism and is produced in large quantities when cells degrade their DNA. Rock and co-workers ${ }^{35}$ showed that crystalline, but not soluble, uric acid has a potent adjuvant effect on dendritic cells. Moreover, depletion of uric acid using allopurinol leads to a dramatic decrease of CD8 T-cell responses to antigens in transplanted syngeneic cells. ${ }^{18}$ The requirement for uric acid as an adjuvant is bypassed by CD40 ligation. The adjuvant role of crystalline uric acid has also been shown in a tumor model. ${ }^{36}$ Tumor regression is associated with high levels of uric acid and peritumoral injection of uric acid accelerated rejection, whereas depletion of uric acid delayed tumor regression. Interestingly, two chemotherapeutic drugs, cyclophosphamide (CY) and etoposide, induce high levels of uric acid levels. ${ }^{36}$ It has been proposed that the requirement for crystalline uric acid serves as a threshold device: only when uric acid levels reach the saturation point, indicating widespread cell stress, will an immune response be initiated. These data have led to a model in which dying tumor cells release uric acid and antigens, thereby stimulating dendritic cells to generate antitumor responses. According to this model, high-level uric acid is a key mediator of immunogenic apoptosis. Such a role for uric acid is consistent with the wellestablished link between monosodium urate, gout and inflammatory responses and also explains the 'tumor lysis syndrome': when successful therapy results in tumor regression, the massive tumor cell apoptosis results in the release of large amounts of uric acid in the circulation, which may trigger (pro-inflammatory) side effects. ${ }^{19,37}$ These findings have led to the question how uric acid is detected by the immune system. Recent data indicate that macrophages harbor a multi-protein complex, referred to as the inflammasome. ${ }^{38}$ The inflammasome links molecular pattern receptors to caspase-1. Once activated, caspase- 1 converts the precursors of three IL- 1 family cytokines, IL-1 $\beta$, IL-18 and IL-33, into their active forms. Caspase- 1 is activated by either of the three different molecular pattern-sensing proteins, NALP1-3. NALP3 mediates caspase-1 activation in response to bacterial ligands, nucleic acids, imidazoquinolines, and interestingly, uric acid. ${ }^{39-41}$ Rock and co-workers have shown that uric acid stimulates gouty inflammation in MyD88- and IL-1R-dependent fashion, leading to a model in which the inflammasome in macrophages responds to uric acid by production of $\mathrm{IL}-1$, which then amplifies the response through the IL-1R and the MyD88 pathway. ${ }^{42}$ Uric acid crystals have also been shown to bind CD14, a phagocyte pattern recognition receptor that interacts with TLR2 and TLR4. ${ }^{43}$ Downstream signaling involved p38 phosphorylation, activation of caspase-1 and production of IL-1 $\beta,{ }^{43}$ consistent with a role of the inflammasome. In vascular smooth muscle cells, uric acid stimulated production of MCP-1 through the MAP kinase pathway, with a critical role for $\mathrm{p}^{38} .^{44}$ Uric acid-induced MCP-1 production depends on ROS, reminiscent of the LPS signaling pathway. Thus, the pro-inflammatory capacities of uric acid appear to be mediated by at least two mechanisms, the NALP3 inflammasome in macrophages and a ROS/p38-dependent pathway in other cells. It will be interesting to determine how dendritic cells, which also respond to uric acid, perceive the signal.

Several of the key molecules in the signaling pathways that activate innate immunity are redox sensitive. Both ASK1 and NF- $\kappa \mathrm{B}$ can be activated by ROS. Oxidative activation of ASK1 is essential for LPS signaling. ${ }^{10,11}$ This suggests that intracellular generation of ROS could provide a powerful, TLRindependent stimulatory signal. Increased ROS production is associated with apoptotic cell death after UV irradiation or treatment with chemotherapeutic drugs. Interestingly, the alkylating DNA cross-linkers melphalan and chlorambucil (which are related to $\mathrm{CY}$ ) trigger strong and sustained production of ROS. ${ }^{45}$ Melphalan treatment results in the upregulation of the co-stimulator B7.1 on drug-treated cells, as well as production of IFN- $\beta$ and TNF- $\alpha,{ }^{46}$ and this has been found to be critically dependent on ROS. ${ }^{47} \mathrm{NF}-\kappa \mathrm{B}$ is an important mediator, because an inhibitor of the $\mathrm{NF}-\kappa \mathrm{B}$ regulator IKK $\gamma$ (also known as NEMO) blocks B7.1 upregulation $^{47}$ (Figure 3). Interestingly, IKK $\gamma /$ NEMO bridges the NF- $\kappa \mathrm{B}$ and IRF3/IRF7 pathways through TBK $1,{ }^{48}$ thus potentially linking the L-PAM-induced response with innate immune activation induced by dsRNA or B-DNA. ROS have also been 
associated with uric acid-induced MCP-1 induction, providing further evidence for a link between intracellular redox status and inflammatory responses. Thus, the production of ROS could be one of the key arbiters of immunogenic cell death. Consistent with this, it was shown that oxidized necrotic allogeneic cancer cells, but not heat-killed cells, were taken up by dendritic cells and induced antitumor CD8 T-cell responses. ${ }^{49}$

The clinical relevance of some of these signaling pathways for decoding the type of cell death is illustrated by the fact that doxorubicin is antagonized by the ASK-1 inhibitor raf- $1 .{ }^{50}$ Doxorubicin is one of the chemotherapy agents recently highlighted for its immunogenic profile. If the ASK-1 pathway is activated in doxorubicin-treated cells this is likely to lead to the increased expression of pro-inflammatory genes. The other important example is $\mathrm{CY}$. The immunostimulatory properties of $\mathrm{CY}$ have been known for decades and have been attributed to its specific depletion of, first, suppressor T cells, ${ }^{51}$ and, more recently, regulatory CD4 CD25 T cells. ${ }^{52}$ However, CY also induces a profound and systemic release of type-I IFN. ${ }^{53}$ As we have seen, the related drug melphalan triggers IFN- $\beta$ release via ROS and NF- $\kappa \mathrm{B},{ }^{54}$ it seems likely that a similar mechanism operates during $C Y$-induced apoptosis. Type-I IFN production after CY treatment results in T-cell activation ${ }^{53}$ and is essential for its antitumor effect. ${ }^{54}$ Although the melphalan data establish a clear link between ROS and IFN- $\beta$ production, it should also be noted that $\mathrm{CY}$ treatment in tumor-bearing mice also results in increased uric acid levels, most likely resulting directly from tumor cell death. Obviously, these two pathways could synergize in the induction of an acquired immune response.

\section{Sensing DNA Damage}

An independent pathway links genotoxic stress directly to the innate immune system. DNA damage causes cells to upregulate their expression of the ligands for NKG2D. ${ }^{55,56}$ NKG2D is a receptor expressed by NK cells and activated CD8 $T$ cells that can trigger a cytotoxic response. In mice, three NKG2D ligands have been described, H60, RAE-1 and MULT-1. Tumor cells often express constitutively high levels of NKG2D ligands, possibly resulting from chronic genotoxic stress. Although NKG2D-mediated rejections have been described, tumors typically evolve various escape strategies. First, in vivo NKG2D ligand downregulation has been described. ${ }^{57}$ Second, chronic exposure of NK cells to NKG2D ligands is detrimental to NK-cell function. ${ }^{58,59}$ However, it is possible that the NKG2D recognition system serves to detect early signs of genotoxic stress in pre-cancerous cells. It is also possible that drug-induced DNA damage leads to NKG2D ligand upregulation in certain tumor cells, or tumor stromal cells, which could then activate NK cells.

p53 is a key tumor suppressor that restricts cellular proliferation after DNA damage. Indeed, many tumors carry p53 mutations. Restoration of functional p53 in tumor cells induced cellular senescence and, importantly, triggered a strong innate immune response against the tumor, involving neutrophils, macrophages and NK cells. ${ }^{60}$ These data clearly reveal a strong link between cellular senescence and inflammatory responses.

\section{Cell Death as Seen by Dendritic Cells}

Although stressed cells may display several pro-inflammatory or NK-cell activating features, it seems likely that the generation of a bona fide immune response against antigens associated with dying cells requires dendritic cells. Dendritic cells sample antigens from other cells and present those to $T$ cells, in a process referred to as antigen cross-presentation. Sampling of apoptotic cells most likely plays a key role in antigen cross-presentation. In animal models, it has been shown that tumor antigens are efficiently cross-presented in the draining lymph nodes suggesting that dendritic cells migrate into the tumor, pick up antigen from (apoptotic) tumor cells and travel back to the lymph node. Tumor cell apoptosis induced by cytotoxic drugs can increase the amount of tumor antigen cross-presentation. ${ }^{61}$ However, increased antigen cross presentation will not necessarily lead to an antitumor immune response. In fact, it has been suggested that the default response to cross-presented antigen is a tolerogenic one. This would be an important safeguard against systemic autoimmunity. It is becoming clear that the presence of PAMPs or DAMPs in the apoptotic cells could provide the signal to change this tolerogenic response into an immunostimulatory one. This has been most convincingly shown for a TLR ligand. Thus, the presence of the TLR3-ligand doublestranded RNA in apoptotic cells triggers TLR3 in the phagocytosing dendritic cells, leading to immune activation. ${ }^{12}$ It is less clear how this operates for endogenous stress ligands, although it has been shown that treatment of dendritic cells with crystalline uric acid results in activation. Furthermore, melphalan or chloramphenicol-killed cells activated phagocytosing dendritic cells, possibly via a nucleic aciddependent mechanism. ${ }^{62}$ The best-studied example of the relationship between apoptotic cell death and dendritic-cell activation is the topoisomerase inhibitor doxorubicin. Kroemer and co-workers showed that doxorubicin-killed cells were taken up by dendritic cells, whereas cells undergoing apoptosis after exposure to mitomycin $\mathrm{C}$ were not phagocytosed. $^{2}$ Moreover, the interaction between doxorubicinkilled cells and dendritic cells resulted in DC activation. It was subsequently shown that doxorubicin and some of its analogues uniquely induced translocation of calreticulin to the plasma membrane, which in turn served as a DC uptake signal. ${ }^{3}$ Thus, doxorubicin-induced cell death could be defined as immunostimulatory, with a key role of calreticulin translocation. One of the questions raised by this work is why mitomycin C-killed cells were not phagocytosed by dendritic cells in the in vitro assays. This finding appears to contrast with the efficient and constitutive in vivo cross-presentation of tumor antigens, even without any therapeutic intervention. Our own data indicated that treatment of tumor-bearing mice with the nucleotide analogue gemcitabine, which causes widespread apoptosis in the tumor, led to increased levels of antigen cross-presentation. ${ }^{61}$ Although it is possible that gemcitabine also induced calreticulin translocation, an alternative explanation is that calreticulin-independent phagocytosis takes place in vivo, possibly involving other DC subsets, and that this existing process was amplified by gemcitabineinduced cell death. Thus, in the case of doxorubicin, we hypothesize that the definition of 'immunogenic cell death' 
involves more than calreticulin translocation, that is, an endogenous danger signal. In fact, our own preliminary data suggest that chemotherapy-induced cell death is associated with dramatically increased IL-6 expression levels. In this sense, the example of gouty inflammation is instructive: uric acid induces the production of IL- $1 \beta$ via the inflammasome in macrophages. ${ }^{18} \mathrm{IL}-1 \beta$, in turn, amplifies the response through MyD88-dependent IL-1R signaling. Given the high macrophage content in many tumors, it is not inconceivable that similar inflammasome activation occurs in the tumor-infiltrating macrophages in chemotherapy-treated tumors.

The potential role of different DC subsets in the detection and decoding of apoptotic cells is illustrated by recent work from Janssen and co-workers ${ }^{63}$. These authors found that apoptotic, but not necrotic, cells efficiently stimulated CD8 T-cell responses in a TLR/IL-1R- and MyD88-independent manner, but that this pathway was operational only in Flt3ligand-derived DCs and not in conventional GM-CSF-treated bone marrow-derived DCs. Type-I IFN was essential for this response. In contrast, Chen et al. ${ }^{42}$ recently showed that MyD88 and the IL-1R were essential for neutrophilic inflammatory responses against dying cells, and that IL- $1 \alpha$ is the key mediator for sterile inflammatory responses triggered by necrotic cells. ${ }^{64}$ Interestingly, these authors showed that this process was independent of HMGB1, which is released from necrotic cells, and any of the TLRs. Finally, Kim and coworkers ${ }^{28}$ showed that apoptotic cells undergoing secondary necrosis stimulated priming of autoimmune T cells in a TLR2dependent manner. Combined, these data stress the emerging insight that apoptosis and necrosis can both be immunogenic or not, depending on the molecular events associated with cell death.

\section{The Fine Line between Inflammation and Immunity}

Inflammation does not by default lead to the type of cellmediated immunity that is often associated with antiviral or antitumor responses, that is, IFN- $\gamma$-producing CD4 and CD8 $\mathrm{T}$ cells. In fact, the relationship between inflammation and immunity, especially in the field of cancer therapy, appears to be a very complex one. A first, obvious, point is that anticancer chemotherapy rarely promotes curative T-cell immunity. In fact, the 'tumor lysis syndrome', resulting from the high levels of pro-inflammatory uric acid released from dying cancer cells, is not associated with curative responses. Clearly, uric acid has the potential to trigger antitumor T-cell responses, but this may not always be the eventual outcome. The many examples of tumor cells producing pro-inflammatory factors, for example, IL-6, have led to the hypothesis that tumors themselves are borne out of chronic inflammation. ${ }^{65,66}$ In this regard, mesothelioma may be prototypic; persisting asbestos fibers trigger continuing inflammation and wound repair in the mesothelial cells lining the pleural cavity, the highly cytotoxic environment eventually leading to the emergence of malignant mesothelioma cells. The view that cancer is not necessarily a disease of immunosuppression is supported by observations that several pro-inflammatory factors can have dual effects on tumor growth, promoting either cures of accelerated growth. Examples include nitric oxide and iNOS activity, ${ }^{67,68}$ COX-2 activation ${ }^{69}$ and MCP-1. ${ }^{70}$ Some tumor cells express TLRs, for example, TLR2 and TLR4, and ligation of these receptors, for example by intratumoral bacterial infection, appears to stimulate tumor growth. ${ }^{16,17}$ The recently described IL-17 secreting subset of CD4 T cells (Th17 cells) may play a pivotal role in this. ${ }^{71}$ Differentiation of Th17 cells is promoted by the paradoxical combination of the pro-inflammatory cytokine IL- 6 and the immunosuppressive TGF- $\beta$. Th17 cells promote chronic inflammation, have been implicated in autoimmunity and, perhaps most importantly, negatively regulate IFN- $\gamma$-producing CD8 T cells. Importantly, mice deficient for the Th17 growth factor IL-23 are resistant to tumor growth. ${ }^{72}$ Thus, it is possible that tumor cells have been selected to survive under inflammatory conditions, as long as specific cytotoxic $T$ cell responses are kept under control. TGF- $\beta$, which is indeed produced by a wide variety of tumor cells, may provide the ideal cytokine to achieve this. Therefore, the challenge for therapy is to translate the spectrum of pro-inflammatory signals into useful antitumor immunity. The fact that, among the 'ambiguous' factors, $\mathrm{NO},{ }^{68} \mathrm{MCP}-1$. ${ }^{70}$ and $\mathrm{IL}-23^{73}$ can be used to drive strong antitumor effects indicates that this can be achieved. For example, exposure to NO sensitizes tumor cells to DC-mediated apoptosis, antigen uptake and cross-presentation. ${ }^{16}$ It seems likely that the translation of a given set of pro- and anti-inflammatory signals towards a specific $T$ cell response happens at the level of the dendritic cell. Indeed, it was recently shown that inflammatory mediators are insufficient for full DC activation and result in the generation of CD4 $\mathrm{T}$ cells that lack helper functions. ${ }^{74}$ Consistent with this, our data show that DC activation using an agonistic anti-CD40 antibody could change the transient antitumor effect of gemcitabine into a curative, CD8 T-celldependent response. ${ }^{61}$ Thus, we will need to understand how DCs integrate the different signals they perceive, how DCs in cancer patients differ from normal DCs, and to what extent the antitumor $\mathrm{T}$ cells are still present in patients or need to be generated through vaccination or adoptive cell transfer. The presence of abundant death-associated danger signals in cancer patients may provide an opportunity once we learn to tune the DCs and provide the T cells.

Zeh and co-workers ${ }^{25}$ have put forward the thoughtprovoking hypothesis that the type of cell death (apoptotic versus necrotic) combined with the nature of the danger signal determines the immunological outcome. Thus, apoptotic cell death with exogenous (pathogen-derived) danger signals would drive a Th1-type response (i.e., IFN- $\gamma$-producing cytotoxic $\mathrm{T}$ cells), whereas necrotic cell death with endogenous danger signals (e.g., uric acid, HMGB1) stimulate an inflammatory response without Th1 involvement. In this sense, chronic inflammation and cancer may be linked by the disorderly, necrotic cell death in tumor cells. ${ }^{19}$ If confirmed, this model predicts that the challenge for immunotherapy is to block the antiapoptotic mechanisms in cancer cells, or the pro-inflammatory cycle, and combine this with Th1-biased therapy.

1. Lake RA, Robinson BW. Immunotherapy and chemotherapy - a practical partnership. Nat Rev Cancer 2005; 5: 397-405.

2. Casares N, Pequignot MO, Tesniere A, Ghiringhelli F, Roux S, Chaput N et al. Caspasedependent immunogenicity of doxorubicin-induced tumor cell death. J Exp Med 2005; 202 1691-1701. 
3. Obeid M, Tesniere A, Ghiringhelli F, Fimia GM, Apetoh L, Perfettini JL et al. Calreticulin exposure dictates the immunogenicity of cancer cell death. Nat Med 2007; 13: 54-61.

4. Skoberne M, Beignon AS, Larsson M, Bhardwaj N. Apoptotic cells at the crossroads of tolerance and immunity. Curr Top Microbiol Immunol 2005; 289: 259-292.

5. Matzinger $P$. The danger model: a renewed sense of self. Science 2002; 296: 301-305

6. Matzinger P. Friendly and dangerous signals: is the tissue in control? Nat Immunol 2007; 8: $11-13$.

7. Akira S. TLR signaling. Curr Top Microbiol Immunol 2006; 311: 1-16.

8. Baccala R, Hoebe K, Kono DH, Beutler B, Theofilopoulos AN. TLR-dependent and TLRindependent pathways of type I interferon induction in systemic autoimmunity. Nat Med 2007; 13: 543-551.

9. Roberts ZJ, Goutagny N, Perera PY, Kato H, Kumar H, Kawai T et al. The chemotherapeutic agent DMXAA potently and specifically activates the TBK1-IRF-3 signaling axis. J Exp Med 2007; 204: 1559-1569.

10. Chiang E, Dang O, Anderson K, Matsuzawa A, Ichijo H, David M. Cutting edge: apoptosisregulating signal kinase 1 is required for reactive oxygen species-mediated activation of IFN regulatory factor 3 by lipopolysaccharide. J Immunol 2006; 176: 5720-5724.

11. Matsuzawa A, Saegusa K, Noguchi T, Sadamitsu C, Nishitoh H, Nagai S et al. ROSdependent activation of the TRAF6-ASK1-p38 pathway is selectively required for TLR4mediated innate immunity. Nat Immunol 2005; 6: 587-592.

12. Schulz O, Diebold SS, Chen M, Naslund TI, Nolte MA, Alexopoulou L et al. Toll-like receptor 3 promotes cross-priming to virus-infected cells. Nature 2005; 433: 887-892.

13. Hiscott J. Another detour on the Toll road to the interferon antiviral response. Nat Struct Mol Biol 2004; 11: 1028-1030.

14. Ruckdeschel K, Pfaffinger G, Haase R, Sing A, Weighardt H, Hacker G et al. Signaling of apoptosis through TLRs critically involves toll//L-1 receptor domain-containing adapter inducing IFN-beta, but not MyD88, in bacteria-infected murine macrophages. J Immunol 2004; 173: 3320-3328.

15. Salaun B, Coste I, Rissoan MC, Lebecque SJ, Renno T. TLR3 can directly trigger apoptosis in human cancer cells. J Immunol 2006; 176: 4894-4901.

16. Huang B, Zhao J, Li H, He KL, Chen Y, Chen SH et al. Toll-like receptors on tumor cells facilitate evasion of immune surveillance. Cancer Res 2005; 65: 5009-5014.

17. Huang B, Zhao J, Shen S, Li H, He KL, Shen GX et al. Listeria monocytogenes promotes tumor growth via tumor cell toll-like receptor 2 signaling. Cancer Res 2007; 67: 4346-4352.

18. Rock KL, Hearn A, Chen CJ, Shi Y. Natural endogenous adjuvants. Springer Semin Immunopathol 2005; 26: 231-246.

19. Zeh III HJ, Lotze MT. Addicted to death: invasive cancer and the immune response to unscheduled cell death. J Immunother (1997) 2005; 28: 1-9.

20. Okabe Y, Kawane K, Akira S, Taniguchi T, Nagata S. Toll-like receptor-independent gene induction program activated by mammalian DNA escaped from apoptotic DNA degradation. J Exp Med 2005; 202: 1333-1339.

21. Ishii KJ, Coban C, Kato H, Takahashi K, Torii Y, Takeshita F et al. A Toll-like receptorindependent antiviral response induced by double-stranded B-form DNA. Nat Immunol 2006; 7: 40-48

22. Cheng G, Zhong J, Chung J, Chisari FV. Double-stranded DNA and double-stranded RNA induce a common antiviral signaling pathway in human cells. Proc Natl Acad Sci USA 2007 104: $9035-9040$.

23. Stetson DB, Medzhitov R. Recognition of cytosolic DNA activates an IRF3-dependent innate immune response. Immunity 2006; 24: 93-103.

24. Bennett RL, Blalock WL, Abtahi DM, Pan Y, Moyer SA, May WS. RAX, the PKR activator sensitizes cells to inflammatory cytokines, serum withdrawal, chemotherapy, and viral infection. Blood 2006; 108: 821-829.

25. Ellerman JE, Brown CK, de Vera M, Zeh HJ, Billiar T, Rubartelli A et al. Masquerader: high mobility group box-1 and cancer. Clin Cancer Res 2007; 13: 2836-2848.

26. Lotze MT, Tracey KJ. High-mobility group box 1 protein (HMGB1): nuclear weapon in the immune arsenal. Nat Rev Immunol 2005; 5: 331-342.

27. Ito N, DeMarco RA, Mailliard RB, Han J, Rabinowich $\mathrm{H}$, Kalinski $\mathrm{P}$ et al. Cytolytic cells induce HMGB1 release from melanoma cell lines. J Leukoc Biol 2007; 81: 75-83.

28. Park JS, Gamboni-Robertson F, He Q, Svetkauskaite D, Kim JY, Strassheim D et al. High mobility group box 1 protein interacts with multiple Toll-like receptors. Am J Physiol Cell Physiol 2006; 290: C917-C924.

29. Apetoh L, Ghiringhelli F, Tesniere A, Obeid M, Ortiz C, Criollo A et al. Toll-like receptor 4dependent contribution of the immune system to anticancer chemotherapy and radiotherapy. Nat Med 2007; 13: 1050-1059.

30. Tian J, Avalos AM, Mao SY, Chen B, Senthil K, Wu H et al. Toll-like receptor 9-dependen activation of DNA-containing immune complexes is mediated by HMGB1 and RAGE. Nat Immunol 2007; 8: 487-496.

31. Schmitt E, Gehrmann M, Brunet M, Multhoff G, Garrido C. Intracellular and extracellula functions of heat shock proteins: repercussions in cancer therapy. J Leukoc Biol 2007; 81 $15-27$.

32. Binder RJ, Srivastava PK. Peptides chaperoned by heat-shock proteins are a necessary and sufficient source of antigen in the cross-priming of CD8+ T cells. Nat Immunol 2005; 6 . 593-599.

33. Castelli C, Rivoltini L, Rini F, Belli F, Testori A, Maio M et al. Heat shock proteins: biological functions and clinical application as personalized vaccines for human cancer. Cancer Immunol Immunother 2004; 53: 227-233.
34. Shi Y, Evans JE, Rock KL. Molecular identification of a danger signal that alerts the immune system to dying cells. Nature 2003; 425: 516-521.

35. Shi Y, Galusha SA, Rock KL. Cutting edge: elimination of an endogenous adjuvant reduces the activation of CD8 T lymphocytes to transplanted cells and in an autoimmune diabetes model. J Immunol 2006; 176: 3905-3908.

36. Hu DE, Moore AM, Thomsen LL, Brindle KM. Uric acid promotes tumor immune rejection. Cancer Res 2004; 64: 5059-5062.

37. Gemici C. Tumour lysis syndrome in solid tumours. Clin Oncol (R Coll Radiol) 2006; 18: 773-780

38. Ogura Y, Sutterwala FS, Flavell RA. The inflammasome: first line of the immune response to cell stress. Cell 2006; 126: 659-662.

39. Kanneganti TD, Ozoren N, Body-Malapel M, Amer A, Park JH, Franchi L et al. Bacterial RNA and small antiviral compounds activate caspase-1 through cryopyrin/Nalp3. Nature 2006; 440: 233-236.

40. Martinon F, Petrilli V, Mayor A, Tardivel A, Tschopp J. Gout-associated uric acid crystals activate the NALP3 inflammasome. Nature 2006; 440: 237-241.

41. Martinon F, Tschopp J. Inflammatory caspases and inflammasomes: master switches of inflammation. Cell Death Differ 2007; 14: 10-22.

42. Chen CJ, Shi Y, Hearn A, Fitzgerald K, Golenbock D, Reed G et al. MyD88-dependent IL-1 receptor signaling is essential for gouty inflammation stimulated by monosodium urate crystals. J Clin Invest 2006; 116: 2262-2271.

43. Scott $P, M a ~ H$, Viriyakosol S, Terkeltaub R, Liu-Bryan R. Engagement of CD14 mediates the inflammatory potential of monosodium urate crystals. J Immunol 2006; 177: 6370 6378 .

44. Kanellis J, Watanabe S, Li JH, Kang DH, Li P, Nakagawa T et al. Uric acid stimulates monocyte chemoattractant protein-1 production in vascular smooth muscle cells via mitogen-activated protein kinase and cyclooxygenase-2. Hypertension 2003; 41: 1287-1293.

45. Gorman A, McGowan A, Cotter TG. Role of peroxide and superoxide anion during tumour cell apoptosis. FEBS Lett 1997; 404: 27-33.

46. Jovasevic VM, Mokyr MB. Melphalan-induced expression of IFN-beta in MOPC-315 tumorbearing mice and its importance for the up-regulation of TNF-alpha expression. $\mathrm{J}$ Immunol 2001; 167: 4895-4901.

47. Donepudi M, Raychaudhuri P, Bluestone JA, Mokyr MB. Mechanism of melphalan-induced B7-1 gene expression in P815 tumor cells. J Immunol 2001; 166: 6491-6499.

48. Zhao T, Yang L, Sun Q, Arguello M, Ballard DW, Hiscott J et al. The NEMO adaptor bridges the nuclear factor-kappaB and interferon regulatory factor signaling pathways. Nat Immunol 2007; 8: 592-600.

49. Chiang CL, Ledermann JA, Rad AN, Katz DR, Chain BM. Hypochlorous acid enhances immunogenicity and uptake of allogeneic ovarian tumor cells by dendritic cells to crossprime tumor-specific T cells. Cancer Immunol Immunother 2006; 55: 1384-1395.

50. Alavi AS, Acevedo L, Min W, Cheresh DA. Chemoresistance of endothelial cells induced by basic fibroblast growth factor depends on Raf-1-mediated inhibition of the proapoptotic kinase, ASK1. Cancer Res 2007; 67: 2766-2772.

51. North RJ, Awwad M. Elimination of cycling CD4+ suppressor T cells with an anti-mitotic drug releases non-cycling CD8+ $T$ cells to cause regression of an advanced lymphoma. Immunology 1990; 71: 90-95.

52. Ghiringhelli F, Menard C, Puig PE, Ladoire S, Roux S, Martin F et al. Metronomic cyclophosphamide regimen selectively depletes CD4+CD25+ regulatory $T$ cells and restores $\mathrm{T}$ and NK effector functions in end stage cancer patients. Cancer Immunol Immunother 2007; 56: 641-648.

53. Schiavoni G, Mattei F, Di Pucchio T, Santini SM, Bracci L, Belardelli F et al. Cyclophosphamide induces type I interferon and augments the number of CD44(hi) T lymphocytes in mice: implications for strategies of chemoimmunotherapy of cancer. Blood 2000; 95: 2024-2030.

54. Mokyr MB, Place AT, Artwohl JE, Valli VE. Importance of signaling via the IFN-alpha/beta receptor on host cells for the realization of the therapeutic benefits of cyclophosphamide for mice bearing a large MOPC-315 tumor. Cancer Immunol Immunother 2006; 55: 459-468.

55. Gasser S, Orsulic S, Brown EJ, Raulet DH. The DNA damage pathway regulates innate immune system ligands of the NKG2D receptor. Nature 2005; 436: 1186-1190.

56. Gasser $\mathrm{S}$, Raulet $\mathrm{DH}$. The DNA damage response arouses the immune system. Cancer Res 2006; 66: 3959-3962.

57. Bui JD, Carayannopoulos LN, Lanier LL, Yokoyama WM, Schreiber RD. IFN-dependent down-regulation of the NKG2D ligand H60 on tumors. J Immunol 2006; 176: 905-913.

58. Wiemann K, Mittrucker HW, Feger U, Welte SA, Yokoyama WM, Spies T et al. Systemic NKG2D down-regulation impairs NK and CD8 T cell responses in vivo. J Immunol 2005; 175: 720-729.

59. Coudert JD, Zimmer J, Tomasello E, Cebecauer M, Colonna M, Vivier E et al. Altered NKG2D function in NK cells induced by chronic exposure to NKG2D ligand-expressing tumor cells. Blood 2005; 106: 1711-1717.

60. Xue W, Zender L, Miething C, Dickins RA, Hernando E, Krizhanovsky V et al. Senescence and tumour clearance is triggered by p53 restoration in murine liver carcinomas. Nature 2007; 445: 656-660.

61. Nowak AK, Lake RA, Marzo AL, Scott B, Heath WR, Collins EJ et al. Induction of tumor cell apoptosis in vivo increases tumor antigen cross-presentation, cross-priming rather than cross-tolerizing host tumor-specific CD8 T cells. J Immunol 2003; 170: 4905-4913. 
62. Rad AN, Pollara G, Sohaib SM, Chiang C, Chain BM, Katz DR. The differential influence of allogeneic tumor cell death via DNA damage on dendritic cell maturation and antigen presentation. Cancer Res 2003; 63: 5143-5150.

63. McBride S, Hoebe K, Georgel P, Janssen E. Cell-associated double-stranded RNA enhances antitumor activity through the production of type I IFN. J Immunol 2006; 177: 6122-6128.

64. Chen CJ, Kono H, Golenbock D, Reed G, Akira S, Rock KL. Identification of a key pathway required for the sterile inflammatory response triggered by dying cells. Nat Med 2007; 13: 851-856.

65. de Visser KE, Coussens LM. The inflammatory tumor microenvironment and its impact on cancer development. Contrib Microbiol 2006; 13: 118-137.

66. de Visser KE, Eichten A, Coussens LM. Paradoxical roles of the immune system during cancer development. Nat Rev Cancer 2006; 6: 24-37.

67. Badn W, Hegardt P, Fellert MA, Darabi A, Esbjornsson M, Smith KE et al. Inhibition of inducible nitric oxide synthase enhances anti-tumour immune responses in rats immunized with IFN-gamma-secreting glioma cells. Scand J Immunol 2007; 65: 289-297.

68. Pelaez B, Campillo JA, Lopez-Asenjo JA, Subiza JL. Cyclophosphamide induces the development of early myeloid cells suppressing tumor cell growth by a nitric oxidedependent mechanism. J Immunol 2001; 166: 6608-6615.
69. DeLong P, Tanaka T, Kruklitis R, Henry AC, Kapoor V, Kaiser LR et al. Use of cyclooxygenase-2 inhibition to enhance the efficacy of immunotherapy. Cancer Res 2003 63: 7845-7852.

70. Nesbit M, Schaider H, Miller TH, Herlyn M. Low-level monocyte chemoattractant protein-1 stimulation of monocytes leads to tumor formation in nontumorigenic melanoma cells. $\mathrm{J}$ Immunol 2001; 166: 6483-6490.

71. Weaver CT, Hatton RD, Mangan PR, Harrington LE. IL-17 family cytokines and the expanding diversity of effector T cell lineages. Annu Rev Immunol 2007; 25 821-852.

72. Langowski JL, Zhang X, Wu L, Mattson JD, Chen T, Smith $\mathrm{K}$ et al. IL-23 promotes tumour incidence and growth. Nature 2006; 442: 461-465.

73. Kaiga T, Sato M, Kaneda H, Iwakura Y, Takayama T, Tahara H. Systemic administration of IL-23 induces potent antitumor immunity primarily mediated through Th1-type response in association with the endogenously expressed IL-12. J Immunol 2007; 178: 7571-7580.

74. Sporri R, Reis e Sousa C. Inflammatory mediators are insufficient for full dendritic cell activation and promote expansion of CD4+ T cell populations lacking helper function. Nat Immunol 2005; 6: 163-170. 